\title{
Links with nontrivial Alexander polynomial which are topologically concordant to the Hopf link
}

\author{
Min Hoon Kim, David Krcatovich, and JungHwan Park \\ School of Mathematics, Korea Institute for Advanced Study, Seoul 02455, Republic of Korea \\ E-mail address: kminhoon@kias.re.kr \\ Rice University, Department of Mathematics, 6100 Main Street, Houston, TX 77005, USA \\ E-mail address: dk27@rice.edu \\ Rice University, Department of Mathematics, 6100 Main Street, Houston, TX 77005, USA \\ E-mail address: jp35@rice.edu
}

\begin{abstract}
We give infinitely many 2-component links with unknotted components which are topologically concordant to the Hopf link, but not smoothly concordant to any 2-component link with trivial Alexander polynomial. Our examples are pairwise non-concordant.
\end{abstract}

\section{Introduction}

Freedman's topological 4-dimensional surgery theory [Fre82b] has a well-known consequence that knots with trivial Alexander polynomial are topologically slice (see [Fre82a, FQ90, GT04]). Inspired by the result of Freedman, Hillman [Hil02, Section 7.6] proposed a surgery program for 2-component links with linking number one. By completing the program of Hillman, Davis Dav06 proved that every 2-component link with linking number 1 which has trivial Alexander polynomial is topologically concordant to the Hopf link. In other words, the Alexander polynomials of knots and links determine their topological concordance type in these cases. Interestingly, Cha, Friedl and Powell CFP14] proved that these two cases are exceptional. Namely, they showed that the link concordance class is not determined by the Alexander polynomial in any other cases.

Based on Donaldson's diagonalization theorem, Casson and Akbulut observed that there are knots with trivial Alexander polynomial which are not smoothly slice (their results are unpublished, see Cochran and Gompf [CG88]). Following this result, smooth concordance of topologically slice knots has been studied extensively using various modern techniques including gauge theory, Heegaard Floer homology and Khovanov homology (for example, see Gom86, End95, MO07, Liv08, HLR12, CHH13, CH15, Hom15, OSS17, HKL16, DV16]). Most of these examples have trivial Alexander polynomial. It was natural to ask whether every topologically slice knot is smoothly concordant to a knot with trivial Alexander polynomial. Hedden, Livingston and Ruberman HLR12 constructed infinitely many topologically slice knots which are not smoothly concordant to any knot with trivial Alexander polynomial. Actually, they showed that their examples are linearly independent in the smooth knot concordance group.

Cha, T. Kim, Ruberman and Strle CKRS12 constructed an infinite family of links with unknotted components which are topologically concordant, but not smoothly concordant, to the Hopf link. By studying satellite operators, Davis and Ray [DR17] constructed another family of links with the same properties. These families of links have trivial Alexander polynomial. (This fact can be checked using C-complexes.) Inspired by the result of Hedden-Livingston-Ruberman [HLR12, it is natural to ask whether there are links with unknotted components which are topologically concordant to the Hopf link but not smoothly concordant to any link with trivial Alexander polynomial. The goal of this paper is to answer that question. 
Theorem A. There exist infinitely many, pairwise non-concordant 2-component links with unknotted components which are topologically concordant to the Hopf link, but not smoothly concordant to any 2-component link with trivial Alexander polynomial.

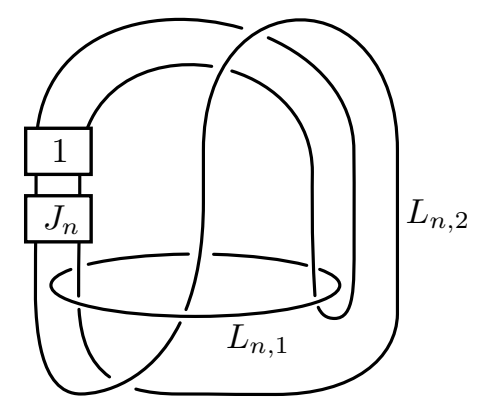

Figure 1. A family of two component links $L_{n}=L_{n, 1} \sqcup L_{n, 2}$.

Our family of examples $L_{n}$ is given in Figure1 1 . It is immediate to see that the components of $L_{n}$ are unknotted. Here the knot $J_{n}$ is

$$
J_{n}=(n D)_{2,4 n-1} \#-T_{2,4 n-1} \# 2(n-1) D
$$

where $D$ is the (positive) Whitehead double of the right handed trefoil. We will see that $J_{n}$ is topologically slice in Lemma 3.19. Assuming that $J_{n}$ is topologically slice, Figure 2 shows that $L_{n}$ is topologically concordant to the Hopf link for any $n$.

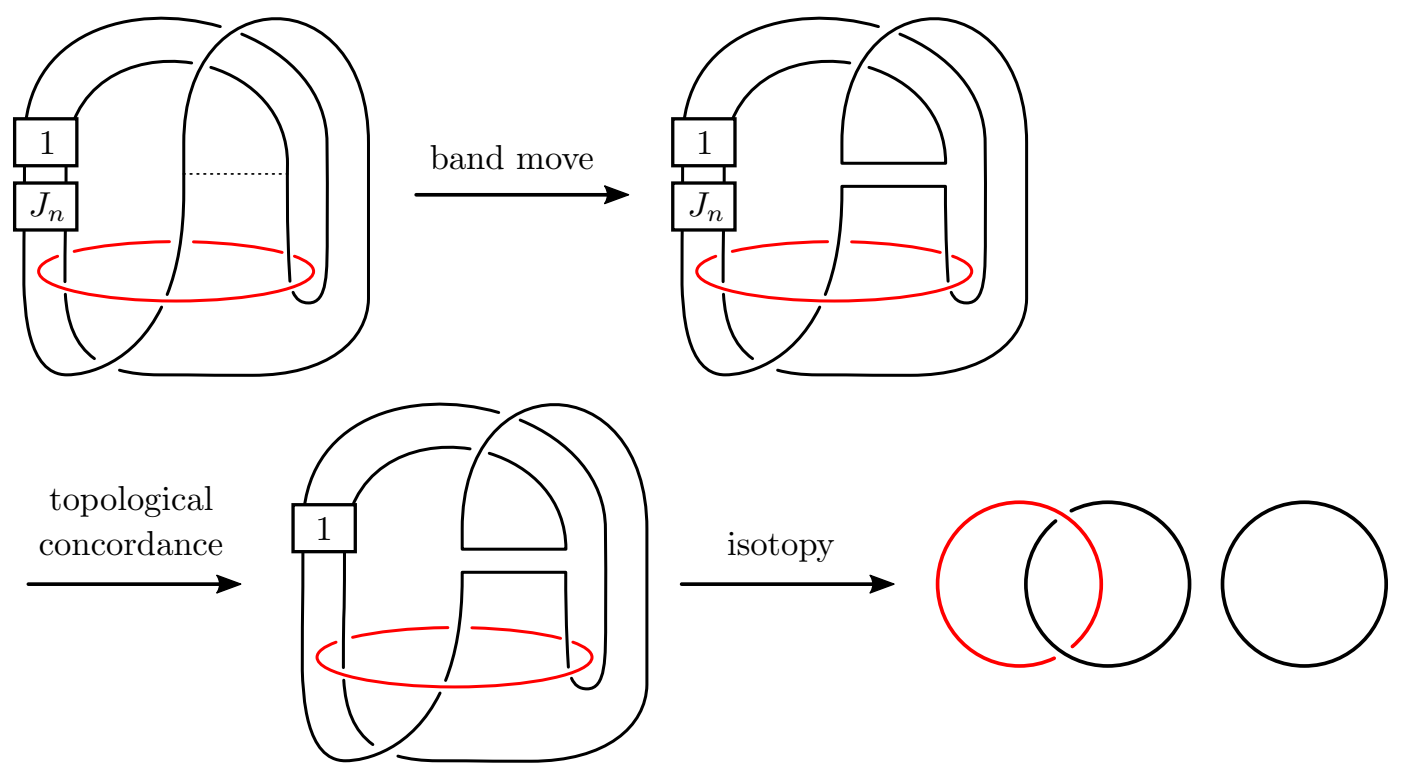

Figure 2. A proof that the link $L_{n}$ is topologically concordant to the Hopf link. The first figure represents $L_{n}$. The second figure is obtained by a band move from the first figure. (The band is depicted as the dotted line in the first figure.) The link in the third figure is topologically concordant to the link in the second figure since $J_{n}$ is topologically slice. It is straightforward to see that the third figure is isotopic to the split union of the Hopf link and the unknot.

The difficult part of Theorem $\mathrm{A}$ is to prove $L_{n}$ is not smoothly concordant to any 2-component link with trivial Alexander polynomial. This part has two ingredients. The first ingredient is the following vanishing theorem which is analogous to an obstruction introduced in [HLR12]. 
Theorem B. Suppose that $L=L_{1} \sqcup L_{2}$ is a 2-component link with linking number 1 . Let $Y_{L}$ be the 3-manifold obtained by doing 1-surgery on $S^{3}$ along $L_{1}$. Denote the image of $L_{2}$ in $Y_{L}$ by $K_{L}$. Let $\Sigma_{K_{L}}$ be the 2-fold cover of $Y_{L}$ branched along $K_{L}$. If $L$ is smoothly concordant to a link $J$ with $\Delta_{J}(s, t)=1$, then the Hedden-Livingston-Ruberman obstruction vanishes for $\Sigma_{K_{L}}$.

For the precise statement of vanishing Hedden-Livingston-Ruberman obstruction, see Theorem 2.8. To prove that $\Sigma_{K_{L_{n}}}$ has non-vanishing Hedden-Livingston-Ruberman obstruction, we will need the following theorem which is based on results of [KP16] and the reduced Floer chain complex introduced by the second author $\operatorname{Krc15}$. Here, $\left\{V_{k}(K)\right\}$ is a sequence of smooth concordance invariants of $K$ which was introduced by Rasmussen Ras03 and then further studied by Ni and Wu NW15. Moreover, Ni and $\mathrm{Wu}$ showed that correction terms of $S_{p / q}^{3}(K)$ can be computed using the sequence $\left\{V_{k}(K)\right\}$. For more details, see Section 3.2 .

Theorem C. Let $J_{n}$ be the knot $(n D)_{2,4 n-1} \#-T_{2,4 n-1} \# 2(n-1) D$ where $D$ is the Whitehead double of the right handed trefoil. For any integer $n \geq 1$, the knot $J_{n}$ is topologically slice and satisfies $V_{0}\left(2 J_{n}\right)=2 n$ and $V_{1}\left(2 J_{n}\right)=2 n-1$.

The rest of the paper is organized as follows. In Section 2, we will show that the Alexander polynomial of $K_{L}$ is determined by the Alexander polynomial of $L$. We will recall the HeddenLivingston-Ruberman obstruction and prove Theorem B. In Section 3, we will recall necessary results on Heegaard Floer invariants of knots and prove Theorem C. In Section 4, we prove Theorem A.

Acknowledgement. This paper was partially written when the authors participated in the conference on knot concordance and 4-manifolds which was held at the Max-Planck-Institut für Mathematik in October 17-21, 2016. The authors would like to thank the Max-Planck-Institut für Mathematik for its stimulating working environment. The first author was partially supported by the Overseas Research Program for Young Scientists through the Korea Institute for Advanced Study. The third author was partially supported by the National Science Foundation grant DMS-1309081. The first and the third authors thank the Hausdorff Institute for Mathematics in Bonn for both support and its outstanding research environment. The authors thank Jae Choon Cha, Matthew Hedden and Daniel Ruberman for comments on the first version of this paper. Finally, we are grateful to the anonymous referee for the detailed and thoughtful suggestions.

\section{A link analogue of the Hedden-Livingston-Ruberman obstruction}

\subsection{Alexander polynomials of knots in homology 3-spheres}

We first recall necessary definitions of the Alexander polynomials of knots and links following Kaw96, Chapter 7] and [Hil02, Chapter 3]. Let $\Lambda$ be a ring which is a unique factorization domain and Nötherian. (In our cases, $\Lambda$ will be either $\mathbb{Z}\left[t^{ \pm 1}\right]$ or $\mathbb{Z}\left[s^{ \pm 1}, t^{ \pm 1}\right]$.) Let $M$ be a finitely generated $\Lambda$-module. Since $M$ is finitely generated, there is an epimorphism $\varphi: \Lambda^{n} \rightarrow M$ for some positive integer $n$. Since $\Lambda$ is Nötherian, we can assume that the kernel of $\varphi$ is generated by $m$ elements with $m \geq n$. There is an $m \times n$ matrix $P$ over $\Lambda$ and an exact sequence

$$
\Lambda^{m} \stackrel{P}{\longrightarrow} \Lambda^{n} \longrightarrow M \longrightarrow 0 .
$$

The matrix $P$ is called a presentation matrix of $M$. The 0 -th characteristic polynomial of $M$, denoted by $\Delta_{0}(M) \in \Lambda$, is the greatest common divisor of the elements of the ideal generated by $n \times n$ minors of $P$. It is known that $\Delta_{0}(M)$ is well-defined up to multiplication by a unit of $\Lambda$. (That is, $\Delta_{0}(M)$ does not depend on the choice of a presentation matrix $P$.)

Remark 2.1. For a given short exact sequence of finitely generated $\Lambda$-modules,

$$
0 \longrightarrow M_{1} \longrightarrow M \longrightarrow M_{2} \longrightarrow 0
$$

we have $\Delta_{0}(M) \doteq \Delta_{0}\left(M_{1}\right) \Delta_{0}\left(M_{2}\right)$ where $\doteq$ denotes the equality up to multiplication by a unit of $\Lambda$ (see [Kaw96, Lemma 7.2.7]). 
For an oriented knot $K$ in a homology 3-sphere $Y$, let $E_{K}=Y-\nu(K)$. Let $\widetilde{E_{K}} \rightarrow E_{K}$ be the infinite cyclic cover induced by the abelianization map $\pi_{1}\left(E_{K}\right) \rightarrow H_{1}\left(E_{K}\right)$. As in [Hil02, Chapter 2], $H_{*}\left(E_{K} ; \mathbb{Z}\left[t^{ \pm 1}\right]\right)$ denotes the homology of $\widetilde{E_{K}}$. We define the Alexander polynomial $\Delta_{K}(t)$ of $K$ to be $\Delta_{0}\left(H_{1}\left(E_{K} ; \mathbb{Z}\left[t^{ \pm 1}\right]\right)\right)$.

Similarly, for a 2-component link $L$ in $S^{3}$, let $E_{L}=S^{3}-\nu(L)$. Let $\widetilde{E_{L}} \rightarrow E_{L}$ be the $\mathbb{Z} \oplus \mathbb{Z}$-cover induced by the abelianization map $\pi_{1}\left(E_{L}\right) \rightarrow H_{1}\left(E_{L}\right)$. We define the Alexander polynomial $\Delta_{L}(s, t)$ of $L$ to be $\Delta_{0}\left(H_{1}\left(E_{L} ; \mathbb{Z}\left[s^{ \pm 1}, t^{ \pm 1}\right]\right)\right)$.

Technically, our definitions of the Alexander polynomials of knots/links seem to be different from the ones given in [Kaw96], but they are in fact equivalent (see [Kaw96, Proposition 7.3.4(2)]).

Definition 2.2 (Knot obtained by 1-surgery on the first component). Let $L=L_{1} \sqcup L_{2}$ be a 2component link in $S^{3}$ with linking number 1. Let $Y_{L}$ be the homology 3-sphere obtained from $S^{3}$ by doing 1-surgery along $L_{1}$. Let $K_{L}$ be the knot which is the image of $L_{2}$ in $Y_{L}$. We say $K_{L}$ is the knot obtained from $L$ by doing 1-surgery on the first component.

For example, the knot $K_{L_{n}}$ drawn in Figure 3 is the knot obtained from $L_{n}$ by doing 1-surgery on the first component. A simple proof of the following lemma was suggested by the anonymous referee.

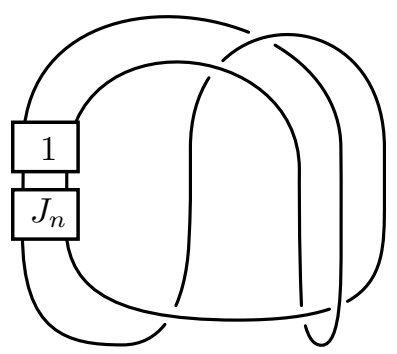

Figure 3. A knot $K_{L_{n}}$ obtained from $L_{n}$ by doing 1-surgery on the first component.

Lemma 2.3. Let $L$ be a 2-component link with linking number 1 . If the Alexander polynomial of $L$ is trivial, then the Alexander polynomial of the knot $K_{L}$ is also trivial.

Proof. Suppose that $L=L_{1} \sqcup L_{2}$ is a 2-component link with linking number 1, and has trivial Alexander polynomial. Let $Y_{L}$ be the homology 3-sphere obtained from $S^{3}$ by doing 1-surgery along $L_{1}$. Consider the link $L^{\prime} \subset Y_{L}$ whose first component is the core of the 1-surgery on $L_{1}$ and second component is the image of $L_{2}$. Note that the second component of $L^{\prime}$ is the knot $K_{L} \subset Y_{L}$.

Since $\Delta_{L}(s, t)=1$, the $\mathbb{Z} \oplus \mathbb{Z}$-cover of the exterior of $L$ has trivial first homology. The Alexander polynomial of $L^{\prime}$ is also trivial because the exteriors of $L$ and $L^{\prime}$ are homeomorphic, so their $\mathbb{Z} \oplus \mathbb{Z}$ covers are homeomorphic. Since the linking number of $L^{\prime}$ is $1, \Delta_{K_{L}}(t)=\Delta_{L^{\prime}}(1, t)=1$ by Torres' condition [Tor53, page 57].

Lemma 2.4. Let $L=L_{1} \sqcup L_{2}$ be a 2-component link with linking number 1 . If $L$ is smoothly concordant to a 2-component link $J$ with trivial Alexander polynomial, then the knots $K_{L}$ and $K_{J}$ are smoothly concordant in a smooth homology $S^{3} \times[0,1]$ and $\Delta_{K_{J}}(t)=1$.

Proof. Suppose that $L$ is smoothly concordant to $J$ with $\Delta_{J}(s, t)=1$ via a smooth concordance $C_{1} \sqcup C_{2} \subset S^{3} \times[0,1]$. Let $Z$ be the result of 1-surgery of $S^{3} \times[0,1]$ along $C_{1}$. Note that $\partial Z=Y_{L} \sqcup-Y_{J}$. By Alexander duality and Mayer-Vietoris sequence, $Z$ is a smooth homology $S^{3} \times[0,1]$. The image of $C_{2}$ in $Z$ gives a smooth concordance between the results of surgery $K_{L}$ and $K_{J}$ in $Z$. By Lemma 2.3 . $\Delta_{K_{J}}(t)=1$.

Remark 2.5. An analogous statement of Lemma 2.4 also holds in the topological category. 


\subsection{Hedden-Livingston-Ruberman obstruction and its link analogue}

Theorem B is inspired by the main obstruction theorem of [HLR12] which we recall in Theorem 2.7 For the reader's convenience, we recall some necessary definitions following [HLR12, Sections 2-3]. For more details, see HLR12.

Let $Y$ be a $\mathbb{Z}_{2}$-homology 3-sphere. Recall that $Y$ has a non-singular $\mathbb{Q} / \mathbb{Z}$-valued linking form

$$
\lambda_{Y}: H_{1}(Y) \times H_{1}(Y) \longrightarrow \mathbb{Q} / \mathbb{Z},
$$

which is the adjoint of the following composition of isomorphisms from Poincaré duality, the Bockstein long exact sequence and the universal coefficient theorem,

$$
H_{1}(Y) \stackrel{\mathrm{PD}}{\longrightarrow} H^{2}(Y) \stackrel{\beta^{-1}}{\longrightarrow} H^{1}(Y ; \mathbb{Q} / \mathbb{Z}) \stackrel{\mathrm{UCT}}{\longrightarrow} \operatorname{Hom}_{\mathbb{Z}}\left(H_{1}(Y), \mathbb{Q} / \mathbb{Z}\right) .
$$

A subgroup $M$ of $H_{1}(Y)$ is called a metabolizer for the linking form $\lambda_{Y}: H_{1}(Y) \rightarrow H_{1}(Y) \rightarrow \mathbb{Q} / \mathbb{Z}$ if $M=M^{\perp}$ where

$$
M^{\perp}=\left\{x \in H_{1}(Y) \mid \lambda_{Y}(x, y)=0 \text { for all } y \in M\right\} .
$$

Definition 2.6. For $z \in H_{1}(Y)$, let $\mathfrak{s}_{z}$ be the unique $\operatorname{Spin}^{c}$ structure of $Y$ which satisfies $c_{1}\left(\mathfrak{s}_{z}\right)=$ $2 \widehat{z} \in H^{2}(Y)$ where $\widehat{z}$ is the Poincaré dual of $z$. In particular, $\mathfrak{s}_{0}$ is the unique Spin structure on $Y$. Define $\bar{d}\left(Y, \mathfrak{s}_{z}\right):=d\left(Y, \mathfrak{s}_{z}\right)-d\left(Y, \mathfrak{s}_{0}\right)$.

We remark that the $\bar{d}$-invariant that we use in this paper is different from the $\bar{d}$-invariant coming from involutive Heegaard Floer homology HM17.

We will use the following theorem which is analogous to [HLR12, Theorem 3.2]. The proof of HLR12, Theorem 3.2] based on [HLR12, Proposition 2.1] can be easily adapted, so we leave the proof of Theorem 2.7 to the reader.

Theorem 2.7. Let $Y$ be a $\mathbb{Z}_{2}$-homology 3 -sphere. If there is a smooth $\mathbb{Z}_{2}$-homology 4-ball $W$ such that $\partial W=Y \# Z$ for some homology 3-sphere $Z$, then there is a metabolizer $M \subset H_{1}(Y)$ for the linking form

such that $\bar{d}\left(Y, \mathfrak{s}_{m}\right)=0$ for all $m \in M$.

$$
\lambda_{Y}: H_{1}(Y) \times H_{1}(Y) \longrightarrow \mathbb{Q} / \mathbb{Z}
$$

Now we prove Theorem $\mathrm{B}$ whose precise version is given in Theorem 2.8

Theorem 2.8. Let $L=L_{1} \sqcup L_{2}$ be a 2-component link with linking number 1 . Let $Y_{L}$ be the 3manifold obtained by doing 1-surgery on $S^{3}$ along $L_{1}$. Denote the image of $L_{2}$ in $Y_{L}$ by $K_{L}$. Let $\Sigma_{K_{L}}$ be the 2-fold cover of $Y_{L}$ branched along $K_{L}$. If $L$ is smoothly concordant to a link with trivial Alexander polynomial, then there is a metabolizer $M$ for the linking form

$$
\lambda_{\Sigma_{K_{L}}}: H_{1}\left(\Sigma_{K_{L}}\right) \times H_{1}\left(\Sigma_{K_{L}}\right) \longrightarrow \mathbb{Q} / \mathbb{Z}
$$

such that $\bar{d}\left(\Sigma_{K_{L}}, \mathfrak{s}_{m}\right)=0$ for all $m \in M$.

Proof. We continue to use notations used in the proof of Lemma 2.4. Suppose that $L$ is concordant to a 2-component link $J$ with trivial Alexander polynomial via a concordance $C_{1} \sqcup C_{2}$. By doing 1-surgery on the first component $C_{1}$ of the concordance, we obtain a concordance $C$ from $K_{L}$ to $K_{J}$ in $Z$ where $Z$ is a $\mathbb{Z}$-homology $S^{3} \times[0,1]$. By Lemma 2.4 the knot $K_{J}$ satisfies $\Delta_{K_{J}}(t)=1$. The double branched cover $\Sigma_{C}$ of $Z$ branched along $C$ is a $\mathbb{Z}_{2}$-homology cobordism from $\Sigma_{K_{L}}$ to $\Sigma_{K_{J}}$. Since $\Delta_{K_{J}}(t)=1, \Sigma_{K_{J}}$ is an integral homology 3 -sphere. By removing an arc in $\Sigma_{C}$ joining $\Sigma_{K_{L}}$ and $\Sigma_{K_{J}}$, we obtain a smooth $\mathbb{Z}_{2}$-homology 4 -ball $W$ whose boundary is $\Sigma_{K_{J}} \#-\Sigma_{K_{L}}$. By applying Theorem 2.7, the conclusion follows.

\section{Computation of $\bar{d}$-invariants}

Our computation of $\bar{d}$-invariants has several ingredients. 


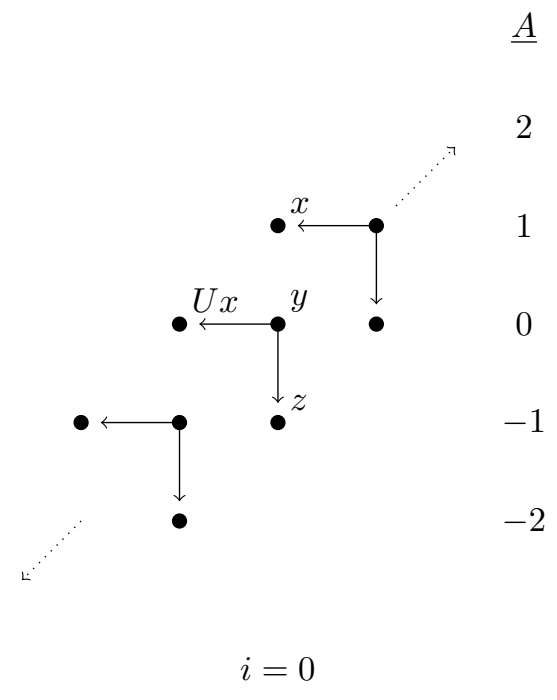

Figure 4. The knot Floer complex of the right-handed trefoil, $C F K^{\infty}\left(T_{2,3}\right)$. It is freely generated over $\mathbb{F}\left[U, U^{-1}\right]$ by $x, y$ and $z$. The differential of both $x$ and $z$ vanishes, while $\partial y=U x+z$. The homological gradings (not shown) are determined by the fact that $M(x)=0, \partial$ decreases $M$ by 1 , and multiplication by $U$ decreases $M$ by 2 .

\subsection{Knot Floer complexes}

Heegaard Floer homology associates a filtered chain complex $C F^{\infty}$ to an appropriate Heegaard diagram for a closed three-manifold OS04b. We call this filtration the algebraic filtration, to distinguish it below. The homology of this and various sub- and quotient complexes are invariants of the threemanifold. From this, Ozsváth and Szabó define a correction term $d(Y, \mathfrak{t}) \in \mathbb{Q}$ associated to a rational homology sphere $Y$ with $\operatorname{Spin}^{c}$ structure $\mathfrak{t}$ OS03.

Ozsváth and Szabó, and independently Rasmussen, showed that a knot $K \subset Y$ can be used to define a second filtration, which we call the Alexander filtration and denote by $A$, yielding a $\mathbb{Z} \oplus \mathbb{Z}$-filtered chain complex $C F K^{\infty}(Y, K)$, defined up to filtered chain homotopy equivalence OS04a, Ras03. In the case of $K \subset S^{3}$, we will simply write $C F K^{\infty}(K)$. We will denote the grading on this complex by $M$. This complex can be used to compute Heegaard Floer homology of surgeries along $K$ OS08, OS11.

We will represent $C F K^{\infty}(K)$ in the $(i, j)$-plane. The complex is finitely generated over $\mathbb{F}\left[U, U^{-1}\right]$, where $U$ is a formal variable, and $\mathbb{F}$ denotes the field with two elements. Each generator $x$ has an Alexander filtration level $A(x)$, and we represent $x$ as a dot at $(0, A(x))$, and, for $i \in \mathbb{Z}$, we represent the homogeneous element $U^{i} x$ as a dot at $(-i, A(x)-i)$ (that is, multiplication by $U$ lowers each of the filtration levels by 1$)$. The differential $\partial\left(U^{i} x\right)$ is a finite sum of homogeneous elements, which we represent by drawing an arrow from $U^{i} x$ to each element. Thus the Alexander filtration is seen vertically in the plane, and the algebraic filtration is seen horizontally; the filtration is manifested by the fact that the arrows must not go up or to the right. See Figure 4 for an example.

Suppose $S$ is a subset of $\mathbb{Z} \oplus \mathbb{Z}$ with the property that

$$
(i, j) \in S \Longrightarrow(i-m, j-n) \in S \text { for all } m, n \geq 0 .
$$

Then the subset of $C$ generated (over $\mathbb{F}$ ) by the elements with $(i, j)$-coordinates in $S$ is a filtered subcomplex, which we will denote $C S$. Thus $C\{i \leq 0\}$ is the subcomplex consisting of everything on or to the left of the $j$-axis, and $C\{i \leq 0, j \leq k\}$ is the "third quadrant" shaped subcomplex, as seen in Figure 5 .

Our interest in knot Floer complexes here will be to compute $d$-invariants of surgeries. Ozsváth and Szabó showed that the Heegaard Floer homology of $S_{p / q}^{3}(K)$ can be computed as the homology 


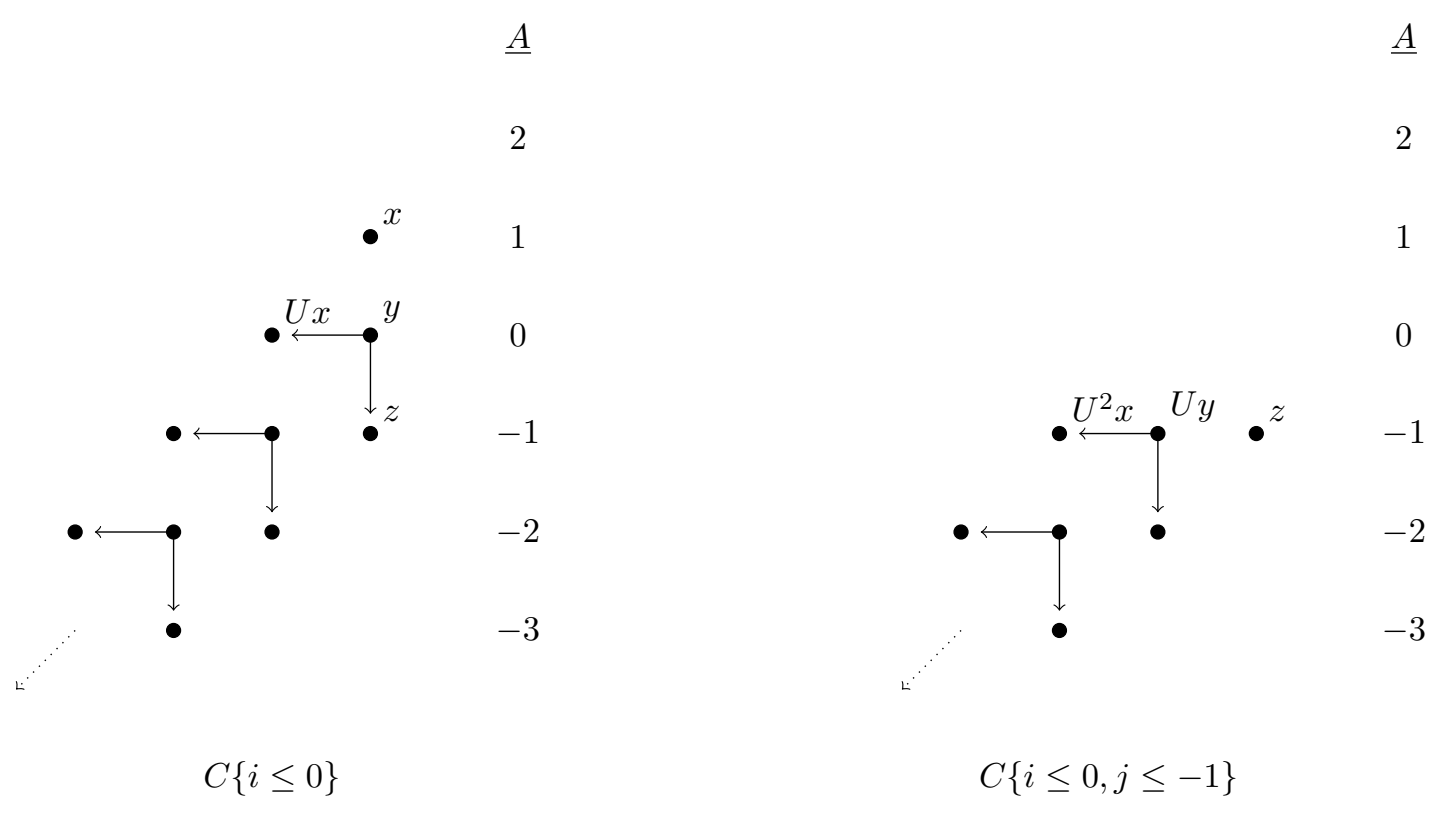

Figure 5. Filtered subcomplexes of $C=C F K^{\infty}\left(T_{2,3}\right)$.

of a mapping cone involving such subcomplexes or their corresponding quotients. See OS11] for full details; we will review what is relevant for our purposes below.

\section{2. $V_{i}$-sequences}

If $C=C F K^{\infty}(K)$ for some knot $K \subset S^{3}$, then $H_{*}(C\{i \leq 0\})$ is isomorphic to a "tower" $\mathcal{T}^{-}$; that is, isomorphic to $\mathbb{F}[U]$, with 1 supported in grading zero.

Let

be inclusion. We define

$$
v_{k}: C\{i \leq 0, j \leq k\} \longrightarrow C\{i \leq 0\}
$$

$$
V_{k}(K):=\max \left\{M(x) \mid\left(v_{k}\right)_{*}(x) \in \mathcal{T}^{-},\left(v_{k}\right)_{*}(x) \neq 0\right\},
$$

where $M$ is the homological grading. Thus, from $C F K^{\infty}(K)$, we get a sequence of nonnegative integers $\left\{V_{k}(K)\right\}$. These were introduced by Rasmussen [Ras03, Definition 7.1 and following discussion] (as "local $h$-invariants"), and then, with the notation here, by Ni and Wu in their study of cosmetic surgeries NW15.

Here we record some useful properties of the $V_{k}$ 's.

Proposition 3.1 ([NW15, Proposition 1.6 and Remark 2.10]). Let $p, q$ be relatively prime integers and $i \in \mathbb{Z}_{p}$. Denote the unknot by $U$. For any knot $K$,

$$
d\left(S_{p / q}^{3}(K), \mathfrak{t}_{i}\right)=d\left(S_{p / q}^{3}(U), \mathfrak{t}_{i}\right)-2 \max \left\{V_{\left\lfloor\frac{i}{q}\right\rfloor}(K), V_{\left\lfloor\frac{p+q-1-i}{q}\right\rfloor}(K)\right\} .
$$

Here $\mathfrak{t}_{i}$ above refers to a specific Spin ${ }^{c}$-structure; see Remark 3.2 for a discussion of this label.

Remark 3.2. For any knot $K$, there is an identification $\varphi: \operatorname{Spin}^{c}\left(S_{p / q}^{3}(K)\right) \rightarrow \mathbb{Z}_{p}$ given in OS11, Sections 4 and 7]. Denote the $\operatorname{Spin}^{c}$ structure of $S_{p / q}^{3}(K)$ which corresponds to $i \in \mathbb{Z}_{p}$ under $\varphi$ by $\mathfrak{t}_{i}$. We recall some facts on $\mathfrak{t}_{i}$ (see [CH15, Appendix B]). The difference between two $\operatorname{Spin}^{c}$ structures of $S_{p / q}^{3}(K)$ is given by the formula

$$
\mathfrak{t}_{j}=\mathfrak{t}_{i}+(j-i) q^{*} \widehat{\mu}
$$


where $q^{*}$ is an integer such that $q q^{*} \equiv 1(\bmod p)$ and $\widehat{\mu} \in H^{2}\left(S_{p / q}^{3}(K)\right)$ is the Poincare dual of the homology class of a meridian of $K$. When $p$ is odd, it is known that the Spin structure of $S_{p / q}^{3}(K)$ is $\mathfrak{t}_{i}$ where $i$ is the $\bmod p$ reduction of the unique element in $\mathbb{Z} \cap\left\{\frac{q-1}{2}, \frac{p+q-1}{2}\right\}$.

Proposition 3.3 ([ $\underline{\operatorname{Ras} 03}$, Proposition 7.6]). For each $k, V_{k}(K)-1 \leq V_{k+1}(K) \leq V_{k}(K)$.

Proposition 3.4 ([BCG17, Proposition 6.1]). For any knots $K, J \subset S^{3}$ and any integers $k$ and $j$,

$$
V_{k+j}(K \# J) \leq V_{k}(K)+V_{j}(J) .
$$

Note that the correction terms of lens spaces which appear in Proposition 3.1 are described by an inductive formula OS03, Proposition 4.8], so the $V_{k}$ 's determine the correction terms of rational surgeries. The following is a consequence of Proposition 3.1 and the fact that $d$ is a $\operatorname{Spin}^{c}$ rational homology cobordism invariant.

Proposition 3.5. For each $k, V_{k}(K)$ is a smooth concordance invariant of $K$.

\section{3. $\nu^{+}$-equivalence}

Following [HW16] and [KP16], we recall the $\nu^{+}$-invariant and $\nu^{+}$-equivalence.

Definition 3.6 ([HW16]). For a knot $K, \nu^{+}(K)$ is the smallest integer $k$ such that $V_{k}(K)=0$.

Proposition 3.7 ([HW16, Proposition 2.3]). The invariant $\nu^{+}$is a smooth concordance invariant. For any knot $K, \nu^{+}(K) \geq 0$ and the equality holds if and only if $V_{0}(K)=0$.

Definition 3.8 ([KP16]). We say two knots $K_{0}$ and $K_{1}$ are $\nu^{+}$-equivalent if

$$
\nu^{+}\left(K_{0} \#-K_{1}\right)=\nu^{+}\left(-K_{0} \# K_{1}\right)=0 \text {. }
$$

Remark 3.9. By Proposition 3.7, two knots $K_{0}$ and $K_{1}$ are $\nu^{+}$-equivalent if and only if

$$
V_{0}\left(K_{0} \#-K_{1}\right)=V_{0}\left(-K_{0} \# K_{1}\right)=0 .
$$

Remark 3.10. Hom's argument in the proof of [Hom17, Proposition 3.11] shows that two knots are $\nu^{+}$-equivalent if and only if $C F K^{\infty}\left(K_{0}\right) \oplus A_{0}$ and $C F K^{\infty}\left(K_{1}\right) \oplus A_{1}$ are filtered chain homotopy equivalent for some acyclic $A_{0}$ and $A_{1}$. (For details, see [KP16, Lemma 3.1].)

Though the following propositions seem to be well-known to experts, we prove them for the reader's convenience.

Proposition 3.11. If $K$ and $J$ are $\nu^{+}$-equivalent, then $V_{i}(K)=V_{i}(J)$ for any $i \geq 0$.

Proof. By Remark 3.9. $V_{0}(K \#-J)=V_{0}(-K \# J)=0$. By Proposition 3.4 and concordance invariance of $V_{i}$, we have

$$
V_{i}(K)=V_{i}(K \#-J \# J) \leq V_{0}(K \#-J)+V_{i}(J)=V_{i}(J) .
$$

(Note that the first equality uses the fact that $-J \# J$ is slice.) The same argument gives the opposite inequality $V_{i}(J) \leq V_{i}(K)$.

Proposition 3.12. Suppose that $K_{i}$ and $J_{i}$ are $\nu^{+}$-equivalent knots for $i=0,1$. Then $K_{0} \# K_{1}$ and $J_{0} \# J_{1}$ are also $\nu^{+}$-equivalent. In particular, if $K$ and $J$ are $\nu^{+}$-equivalent, then $n K$ and $n J$ are $\nu^{+}$-equivalent for any integer $n$.

Proof. By Remark 3.9, $V_{0}\left(K_{i} \#-J_{i}\right)=V_{0}\left(-K_{i} \# J_{i}\right)=0$ for $i=0,1$. By Proposition 3.4, we have

$$
V_{0}\left(K_{0} \# K_{1} \#-J_{0} \#-J_{1}\right) \leq V_{0}\left(K_{0} \#-J_{0}\right)+V_{0}\left(K_{1} \#-J_{1}\right)=0 .
$$

A similar argument shows that $V_{0}\left(-K_{0} \#-K_{1} \# J_{0} \# J_{1}\right)=0$. By Remark 3.9 , the two knots $K_{0} \# K_{1}$ and $J_{0} \# J_{1}$ are also $\nu^{+}$-equivalent.

Now we give some known example of $\nu^{+}$-equivalences. 
Example 3.13. Let $T_{p, q}$ be the $(p, q)$-torus knot and $D$ be the (positive) Whitehead double of the trefoil knot. Then, for any integer $n \geq 1$, both $n D$ and $n T_{2,3}$ are $\nu^{+}$-equivalent to $T_{2,2 n+1}$ by Proposition 6.1 and Theorem B.1 of [HKL16] and Remark 3.10. (Compare [KP16, Example 3.2].)

It is shown in $\mathrm{KP} 16$ ] that $\nu^{+}$-equivalence is preserved under any satellite operation with non-zero winding number. In particular, $\nu^{+}$-equivalence is preserved under cabling operations.

Theorem 3.14 ([KP16, Theorem B]). Suppose that $P \subset S^{1} \times D^{2}$ is a pattern with non-zero winding number. If two knots $K_{0}$ and $K_{1}$ are $\nu^{+}$-equivalent, then $P\left(K_{0}\right)$ and $P\left(K_{1}\right)$ are $\nu^{+}$-equivalent.

Remark 3.15. Note that $V_{k}$ and therefore $\nu^{+}$are invariants of the filtered chain homotopy type of $C F K^{\infty}(K)$. They can likewise be defined for any abstract infinity complex $C$, without knowing whether it is realized by an actual knot (see [HW14, Definition 6.1]). We say two such complexes $C$ and $C^{\prime}$ are $\nu^{+}$-equivalent if

$$
\nu^{+}\left(C \otimes\left(C^{\prime}\right)^{*}\right)=\nu^{+}\left(C^{\prime} \otimes C^{*}\right)=0,
$$

where * denotes the dual complex. We will abuse notation slightly further and say that a complex $C$ and a knot $K$ are $\nu^{+}$-equivalent if $C$ and $C F K^{\infty}(K)$ are.

\subsection{Reduced knot Floer complexes}

The proof of Theorem C will involve computing the $V_{k}$ 's for a connect sum of knots. We discuss here how that can be done effectively when the summands are $\left(\nu^{+}\right.$-equivalent to $L$-space knots or their mirrors, using the reduced knot Floer complex of the second author. We refer the reader to Krc15] for the definition in general, and here review the special case of an $L$-space knot.

By [OS05, Theorem 1.2] and [Hom14, Remark 6.6], when $K$ is an $L$-space knot, we have

$$
C F K^{\infty}(K) \cong \mathbb{F}\left[U, U^{-1}\right] \otimes S_{\left(a_{1}, \ldots, a_{n}\right)},
$$

where $S_{\left(a_{1}, \ldots, a_{n}\right)}$ is a staircase complex, generated by $x_{1}, \ldots, x_{2 n+1}$. Here $x_{2 i+1}$ has filtration level

$$
\left(a_{1}+\cdots+a_{i}, a_{1}+\cdots+a_{n-i}\right),
$$

and $x_{2 i}$ has filtration level

$$
\left(a_{1}+\cdots+a_{i}, a_{1}+\cdots+a_{n-i+1}\right),
$$

where each $a_{i}$ is a positive integer. The differential is given by

$$
\partial\left(x_{2 i+1}\right)=0, \quad \partial\left(x_{2 i}\right)=x_{2 i-1}+x_{2 i+1} .
$$

See Figure 6 for an example. In the case that this complex belongs to a knot $K, g(K)=\sum_{i} a_{i}$ is the Seifert genus of the knot. Note that all the generators with odd indices are pairwise homologous, and any of them generates the homology of the staircase complex. The list $\left(a_{1}, \ldots, a_{n}\right)$ is related to the Alexander polynomial of $K$ as follows. All nonzero terms in $\Delta_{K}(t)$ have coefficients \pm 1 , with signs alternating, and the highest degree term positive. The number $a_{i}$ is the difference between the $(2 i-1)$ th and the $2 i$ th exponent. Because $\Delta_{K}(t)$ is symmetric, this list determines $\Delta_{K}(t)$, so we will say the list corresponds to $\Delta_{K}(t)$, or further that the list corresponds to $K$.

Given an $L$-space knot $K$, let $C=C F K^{\infty}(K)\{i \leq 0\}$. Then the reduced complex $C F K^{-}(K)$ consists merely of the generators in $C$ which have no outgoing nor incoming horizontal arrows, and has trivial differential (see [Krc15, Corollary 4.2]). As a complex, $C F K^{-}(K) \cong \mathbb{F}[U]$, with 1 supported in grading zero. The Alexander filtration descends to a filtration on the reduced complex. The complex no longer has an algebraic filtration, but still has the structure of an $\mathbb{F}[U]$-module, with multiplication by $U$ taking the generator in grading $2 i$ to the generator in grading $2 i-2$.

Remark 3.16. The (Alexander) filtration on the reduced complex of an $L$-space knot can be determined explicitly from the list $\left(a_{1}, a_{2}, \ldots, a_{n}\right)$. If $x$ is the generator (over $\left.\mathbb{F}[U]\right)$ of $\underline{C F K^{-}}(K)$, then $x$ has filtration level $g(K)=a_{1}+\cdots+a_{n}$, and if

$$
a_{1}+\cdots+a_{i} \leq j<a_{1}+\cdots+a_{i+1},
$$

then $U^{j} x$ has filtration level

$$
a_{1}+\cdots+a_{n-i}-j .
$$

For $j \geq g(K), U^{j} x$ has filtration level $-j$. 


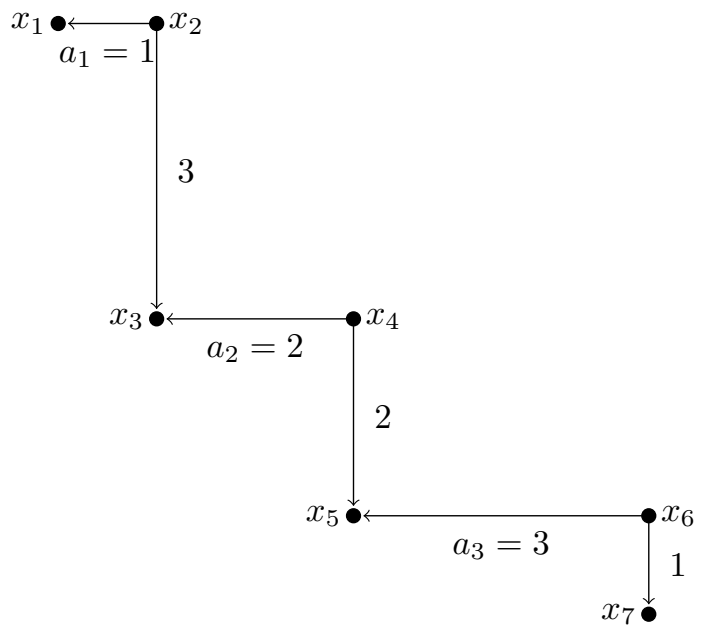

Figure 6 . The staircase $S_{(1,2,3)}$, which generates $C F K^{\infty}\left(T_{4,5}\right)$ over $\mathbb{F}\left[U, U^{-1}\right]$. The generator $x_{1}$ is in filtration level $(0,6)$. The $a_{i}$ 's are the lengths (from left to right) of the horizontal arrows, and also (from bottom to top) of the vertical arrows.

We can compute the $V_{k}$ 's from the reduced complex $\underline{C}$ as

$$
\left.V_{k}(K)=-\frac{1}{2} \max \text { grading of a non } U \text {-torsion generator of } H_{*}(\underline{C}\{j \leq k\})\right\} .
$$

For $K$ an $L$-space knot, $\underline{C}$ is isomorphic to its homology, but Equation 3.2 holds for all knots, where this is not in general the case.

Proposition 3.17 ([Krc15, Theorem 3.4]). If $C$ is a complex with reduction $\underline{C}$, then there is a $(\mathbb{Z}, U)$-filtered chain homotopy equivalence between $C \otimes C F K^{-}\left(K_{2}\right)$ and $\underline{C} \otimes C F K^{-}\left(K_{2}\right)$ for any knot $K_{2}$. In particular, if $C$ is $\nu^{+}$-equivalent to $C F K^{\infty}\left(K_{1}\right)$ for some knot $K_{1}$, then $V_{k}\left(K_{1} \# K_{2}\right)$ can be computed from $\underline{C} \otimes C F K^{-}\left(K_{2}\right)$ using 3.2 .

\subsection{Staircases and connect sums}

The staircases corresponding to two $L$-space knots can be combined to produce a "representative staircase", which can then be used to compute the $V_{k}$ 's of the connect sum of the two knots. This idea is introduced in [BL14, Section 5.1], see also [Krc15, Example 2], GM16, Section 7]. Here we prove the following statement, which is stronger where it applies, as it will in our case of interest.

Lemma 3.18. If $K$ and $J$ are $L$-space knots whose staircase shapes admit a compatible riffle, then

$$
C F K^{\infty}(K \# J) \cong\left(\mathbb{F}\left[U, U^{-1}\right] \otimes S^{\#}\right) \oplus A,
$$

where $S^{\#}$ is the staircase complex corresponding to the riffled shape, and $A$ is an acyclic subcomplex. In particular, $C F K^{\infty}(K \# J)$ is $\nu^{+}$-equivalent to a staircase complex.

The $S^{\#}$ above will be called the representative staircase for the sum. Before proving, we introduce the necessary terminology.

A riffle of two ordered lists $A$ and $B$ is an ordering of $A \cup B$ which restricts to the given orderings on each sublist $A$ and $B$. In a riffle of $A$ and $B$, the opposite successor of an element $a \in A$ (respectively $b \in B$ ) is the first element of $B$ (resp. $A$ ) which appears after $a$ (resp. b), if such an element exists. As an example,

$$
(1,2,3, a, b, 4, c, 5, d, e)
$$

is a riffle of $(1,2,3,4,5)$ and $(a, b, c, d, e)$; the opposite successor of 1 is $a$, the opposite successor of $a$ is 4 , and $d$ and $e$ have no opposite successor. 


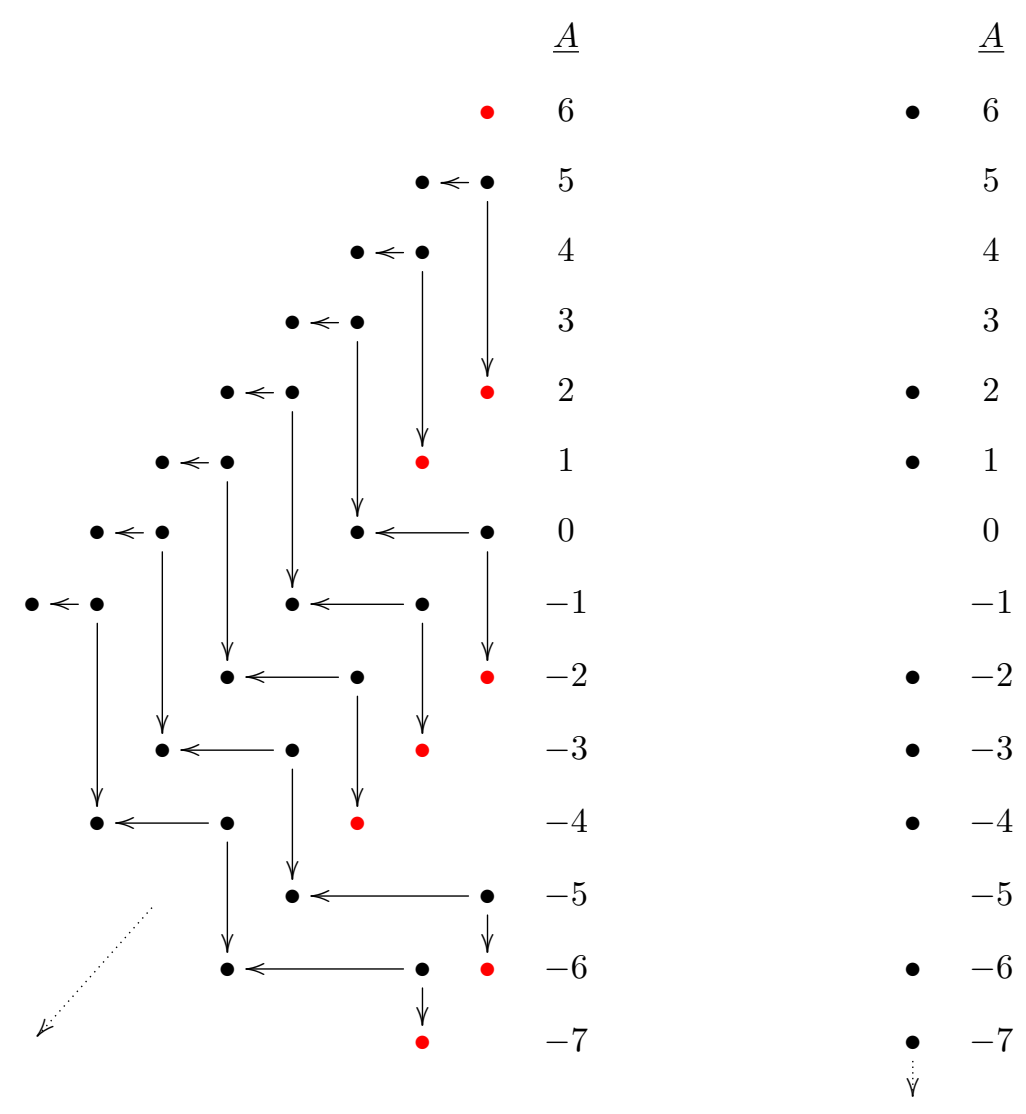

Figure 7. At the left is $C F K^{\infty}\left(T_{4,5}\right)\{i \leq 0\}$, and at the right is the corresponding reduced complex, which consists of the elements colored red on the left; those with no incoming or outgoing horizontal arrows. Multiplication by $U$ in the reduced complex takes one dot downward to the next.

Given a staircase with list $\left(a_{1}, \ldots, a_{n}\right)$, we define the staircase shape as the ordered list of ordered pairs

$$
\left(\left(a_{1}, a_{n}\right),\left(a_{2}, a_{n-1}\right), \ldots,\left(a_{n}, a_{1}\right)\right) .
$$

Two staircase shapes are compatible if thye can be riffled so that if $\left(b_{j}, b_{m-j+1}\right)$ is the opposite successor of $\left(a_{i}, a_{n-i+1}\right)$, then $a_{i} \leq b_{j}$ and $a_{n-i+1} \geq b_{m-j+1}$ (and likewise, if $\left(a_{i}, a_{n-i+1}\right)$ is the opposite successor of $\left(b_{j}, b_{m-j+1}\right)$, then $a_{i} \geq b_{j}$ and $\left.a_{n-i+1} \leq b_{m-j+1}\right)$. We will also call such a riffle compatible.

Proof of Lemma 3.18. Let us assume that $C F K^{\infty}(K) \cong \mathbb{F}\left[U, U^{-1}\right] \otimes S_{\left(a_{1}, \ldots, a_{n}\right)}$, with generators $x_{1}$ through $x_{2 n+1}$, and that $C F K^{\infty}(J) \cong \mathbb{F}\left[U, U^{-1}\right] \otimes S_{\left(b_{1}, \ldots, b_{m}\right)}$, with generators $y_{1}$ through $y_{2 m+1}$. Thus $C F K^{\infty}(K \# J) \cong C F K^{\infty}(K) \otimes C F K^{\infty}(J)$ has basis $x_{i} y_{j}$ (for readability, we write these elements without the ' $\otimes$ ' symbol).

We will choose a new basis for $C F K^{\infty}(K \# J)$ which exhibits the direct sum splitting. The compatibility condition will be precisely what we need to ensure that our change of basis is filtered. Since the staircases are compatible, we can find a riffle of the staircase shapes which is compatible; fix one such riffle. The first generator of the representative staircase will be $x_{1} y_{1}$. Suppose the $(2 k-1)$ th generator on the staircase is $x_{2 i+1} y_{2 k-2 i-1}$, for some $i$. Then, the $k$ th element in the compatible riffle is either

(i) $\left(a_{i+1}, a_{n-i}\right)$ or 
(ii) $\left(b_{k-i}, b_{m-(k-i)+1}\right)$.

In case (i), the next step on the staircase consists of the generators $x_{2 i+2} y_{2 k-2 i-1}$ and $x_{2 i+3} y_{2 k-2 i-1}$. Further, we make the change of basis

$$
\begin{aligned}
x_{2 i+1} y_{2 k-2 i} & \mapsto x_{2 i+2} y_{2 k-2 i-1}+x_{2 i+1} y_{2 k-2 i}, \\
x_{2 i+1} y_{2 k-2 i+1} & \mapsto x_{2 i+3} y_{2 k-2 i-1}+x_{2 i+1} y_{2 k-2 i+1} .
\end{aligned}
$$

In case (ii), the next step in the staircase consists of $x_{2 i+1} y_{2 k-2 i}$ and $x_{2 i+1} y_{2 k-2 i+1}$. We make the filtered change of basis

$$
\begin{aligned}
& x_{2 i+2} y_{2 k-2 i-1} \mapsto x_{2 i+2} y_{2 k-2 i-1}+x_{2 i+1} y_{2 k-2 i}, \\
& x_{2 i+3} y_{2 k-2 i-1} \mapsto x_{2 i+3} y_{2 k-2 i-1}+x_{2 i+1} y_{2 k-2 i+1}
\end{aligned}
$$

This process terminates when the final step of the staircase includes the generator $x_{2 n+1} y_{2 m+1}$.

We now have a collection of acyclic subcomplexes

$$
A_{i, j}:=\left\{x_{2 i} y_{2 j}, x_{2 i-1} y_{2 j}+x_{2 i} y_{2 j-1}, x_{2 i+1} y_{2 j}+x_{2 i} y_{2 j+1}, x_{2 i-1} y_{2 j+1}+x_{2 i+1} y_{2 j-1}\right\},
$$

for $1 \leq i \leq n, 1 \leq j \leq m$.

We have also constructed a subcomplex which is a staircase $S^{\#}$ whose corresponding list is a riffle of $\left(a_{1}, \ldots, a_{n}\right)$ and $\left(b_{1}, \ldots, b_{m}\right)$. Now we have

$$
C F K^{\infty}(K \# J) \cong \mathbb{F}\left[U, U^{-1}\right] \otimes\left(S^{\#} \oplus \bigoplus_{i, j} A_{i, j}\right),
$$

which has the desired form. Figures 8 and 9 provide an illustration of this construction.

Note that such a change of basis can be performed on any tensor product of staircase complexes, but it may not always be filtered - the compatibility assumption ensures that this is indeed a filtered change of basis, and the result can still be used to compute $V_{k}$ 's. The final statement then follows from Hom17, Proposition 3.11].

\subsection{Proof of Theorem $\mathrm{C}$}

Now we prove Theorem $\mathrm{C}$.

Lemma 3.19. Let $D$ be the Whitehead double of the right handed trefoil knot. For any integer $n \geq 1$, let $J_{n}=(n D)_{2,4 n-1} \#-T_{2,4 n-1} \# 2(n-1) D$. Then, the knot $J_{n}$ satisfies the following properties.

(1) $J_{n}$ is topologically slice.

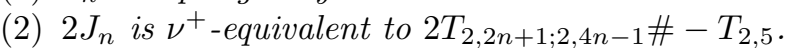

(3) $V_{i}\left(2 J_{n}\right)=V_{i}\left(2 T_{2,2 n+1 ; 2,4 n-1} \#-T_{2,5}\right)$ for any $i$.

Proof. (1) Since $n D$ has trivial Alexander polynomial, it is topologically slice by Freedman [Fre82b]. For any topologically slice knot $J, J_{p, q} \#-T_{p, q}$ is topologically slice. In particular, $(n D)_{2,4 n-1} \#-$ $T_{2,4 n-1}$ is topologically slice for any $n$. As a connected sum of two topologically slice knots, $J_{n}$ is topologically slice for any $n$.

(2) By Example 3.13 for any $n \geq 1, n D,-T_{2,4 n-1}$ and $2(n-1) D$ are $\nu^{+}$-equivalent to $T_{2,2 n+1}$, $-(2 n-1) T_{2,3}$ and $2(n-1) T_{2,3}$, respectively. Since $n D$ is $\nu^{+}$-equivalent to $T_{2,2 n+1},(n D)_{2,4 n-1}$ is $\nu^{+}$-equivalent to $T_{2,2 n+1 ; 2,4 n-1}$ by Theorem 3.14. By Proposition 3.12, $J_{n}$ is $\nu^{+}$-equivalent to $T_{2,2 n+1 ; 2,4 n-1} \#-T_{2,3}$, and hence $2 J_{n}$ is $\nu^{+}$-equivalent to $2 T_{2,2 n+1 ; 2,4 n-1} \#-T_{2,5}$ by Proposition 3.12 and Example 3.13

(3) This follows from the item $(2)$ by Proposition 3.11

By Lemma 3.19, we know $J_{n}$ is topologically slice for every $n$. To prove Theorem C, it suffices to prove Theorem 3.20 .

Theorem 3.20. For any $n \geq 1, V_{0}\left(2 J_{n}\right)=2 n$ and $V_{1}\left(2 J_{n}\right)=2 n-1$. 


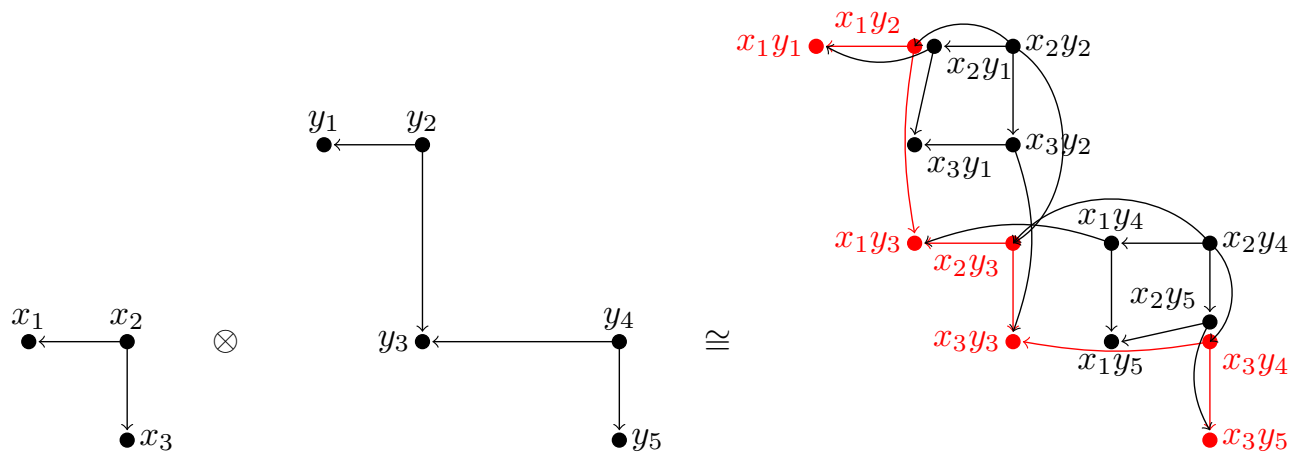

Figure 8. $T_{2,3}$ has staircase shape $((1,1))$, and $T_{3,4}$ has staircase shape $((1,2),(2,1))$. Generating sets for $C F K^{\infty}$ of each are shown on the left, and a generating set for the tensor product complex $C F K^{\infty}\left(T_{2,3}\right) \otimes C F K^{\infty}\left(T_{3,4}\right)$ is shown on the right. Note that the staircase shapes are compatible, with compatible riffle $((1,2),(1,1),(2,1))$, and this is the staircase shape of the subcomplex colored red.

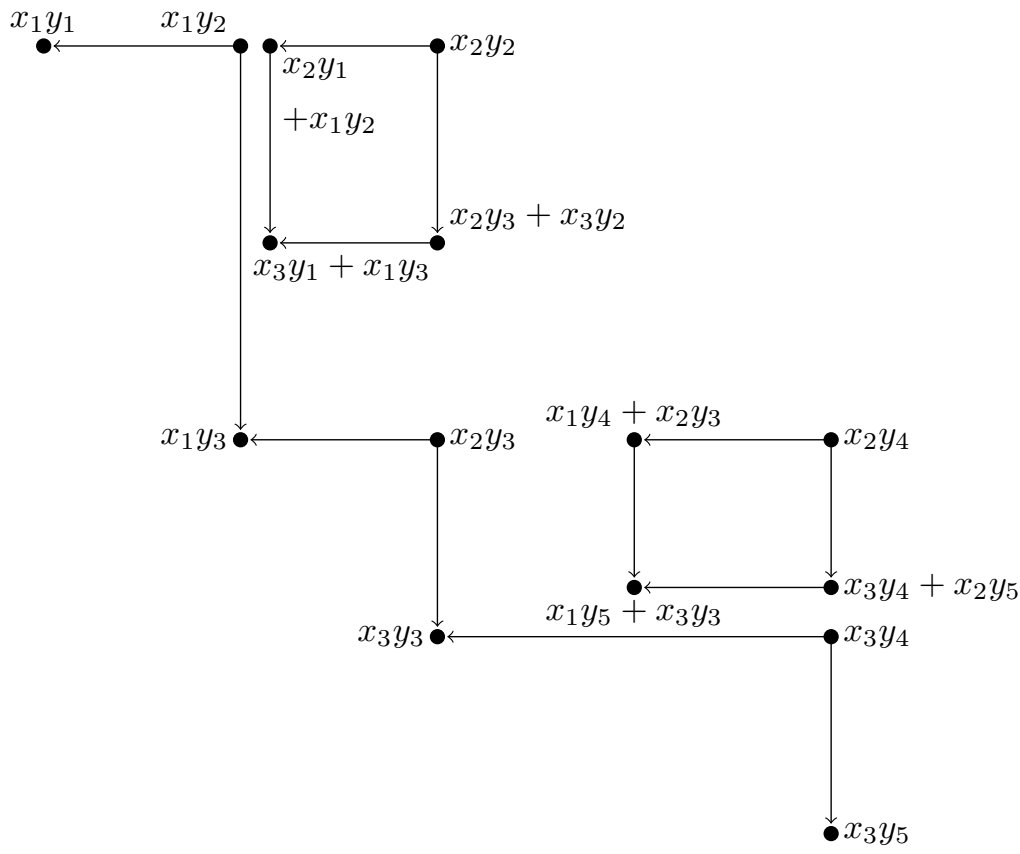

Figure 9. After a filtered change of basis, $C F K^{\infty}\left(T_{2,3} \# T_{3,4}\right)$ is seen to be generated by a direct sum of the staircase $S^{\#}$ (the subcomplex which was colored red in Figure 8) and acyclic complexes.

Proof. It suffices to prove that $V_{0}\left(2 K_{n} \#-T_{2,5}\right)=2 n$ and $V_{1}\left(2 K_{n} \#-T_{2,5}\right)=2 n-1$ by Lemma $3.19(3)$ where $K_{n}=T_{2,2 n+1 ; 2,4 n-1}$. By [Hed09, Theorem 1.10], $K_{n}$ is an $L$-space knot. We have that

$$
\begin{aligned}
\Delta_{K_{n}}(t) & =\Delta_{T_{2,2 n+1}}\left(t^{2}\right) \cdot \Delta_{T_{2,4 n-1}}(t) \\
& =\left(\sum_{i=0}^{2 n}(-1)^{i}\left(t^{2}\right)^{i}\right)\left(\sum_{j=0}^{4 n-2}(-1)^{j} t^{j}\right) \\
& =\sum_{i=0}^{n-1}\left(t^{4 i}-t^{4 i+2}\right)\left(\sum_{j=0}^{4 n-2}(-1)^{j} t^{j}\right)+t^{4 n}\left(\sum_{j=0}^{4 n-2}(-1)^{j} t^{j}\right) \\
& =\sum_{i=0}^{n-1}\left(t^{4 i}-t^{4 i+1}+t^{4 n+4 i-1}-t^{4 n+4 i}\right)+\sum_{j=0}^{4 n-2}(-1)^{j} t^{j+4 n} .
\end{aligned}
$$




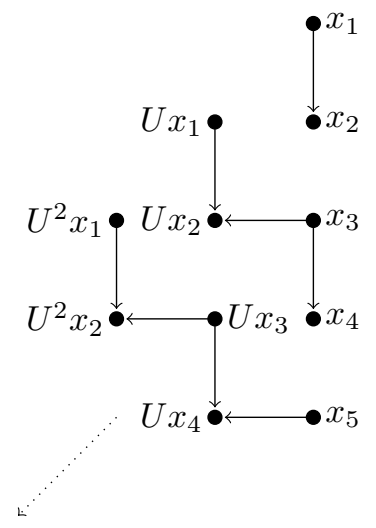

Figure 10. $C F K^{-}\left(-T_{2,5}\right)$.

Note for convenience that, because Alexander polynomials are symmetric, this polynomial is determined by its coefficients up to degree $\frac{1}{2}(8 n-2)=4 n-1$. Therefore, if we let

then we see that

$$
p_{n}(t)=\sum_{i=0}^{n-1} t^{4 i}-t^{4 i+1}
$$

For example, when $n=3$, we have

$$
\Delta_{K_{n}}(t)=p_{n}(t)+t^{4 n-1}+t^{8 n-2} p_{n}\left(t^{-1}\right) .
$$

$$
p_{3}(t)=1-t+t^{4}-t^{5}+t^{8}-t^{9}
$$

SO

$$
\Delta_{K_{3}}(t)=1-t+t^{4}-t^{5}+t^{8}-t^{9}+t^{11}-t^{13}+t^{14}-t^{17}+t^{18}-t^{21}+t^{22} .
$$

The polynomial $\Delta_{K_{n}}(t)$ defines a staircase complex with corresponding list

Thus, the staircase shape is

$$
(\overbrace{1, \ldots, 1}^{n}, 2, \underbrace{3, \ldots, 3}_{n-1})
$$

$$
(\overbrace{(1,3), \ldots,(1,3)}^{n-1},(1,2),(2,1), \underbrace{(3,1), \ldots,(3,1)}_{n-1}) .
$$

It is easily seen that a compatible riffle of the shapes for two copies of $K_{n}$ is given by

$$
(\overbrace{(1,3), \ldots,(1,3)}^{2 n-2},(1,2),(1,2),(2,1),(2,1),(\underbrace{(3,1), \ldots,(3,1)}_{2 n-2}) .
$$

So, by Lemma 3.18, the representative staircase for $K_{n} \# K_{n}$ has list

$$
(\overbrace{1, \ldots, 1}^{2 n}, 2,2, \underbrace{3, \ldots, 3}_{2 n-2}) .
$$

Up to $\nu^{+}$-equivalence then, we can replace $C F K^{\infty}\left(2 K_{n}\right)$ with the staircase complex corresponding to this list, which we will denote by $C_{n}$.

The torus knot $T_{2,5}$ is also an $L$-space knot, which corresponds to the list $(1,1)$. For brevity, we let $C^{-}$denote the complex for the mirror image, $C F K^{-}\left(-T_{2,5}\right)$. A basis for $C^{-}$is given in Figure 10. 


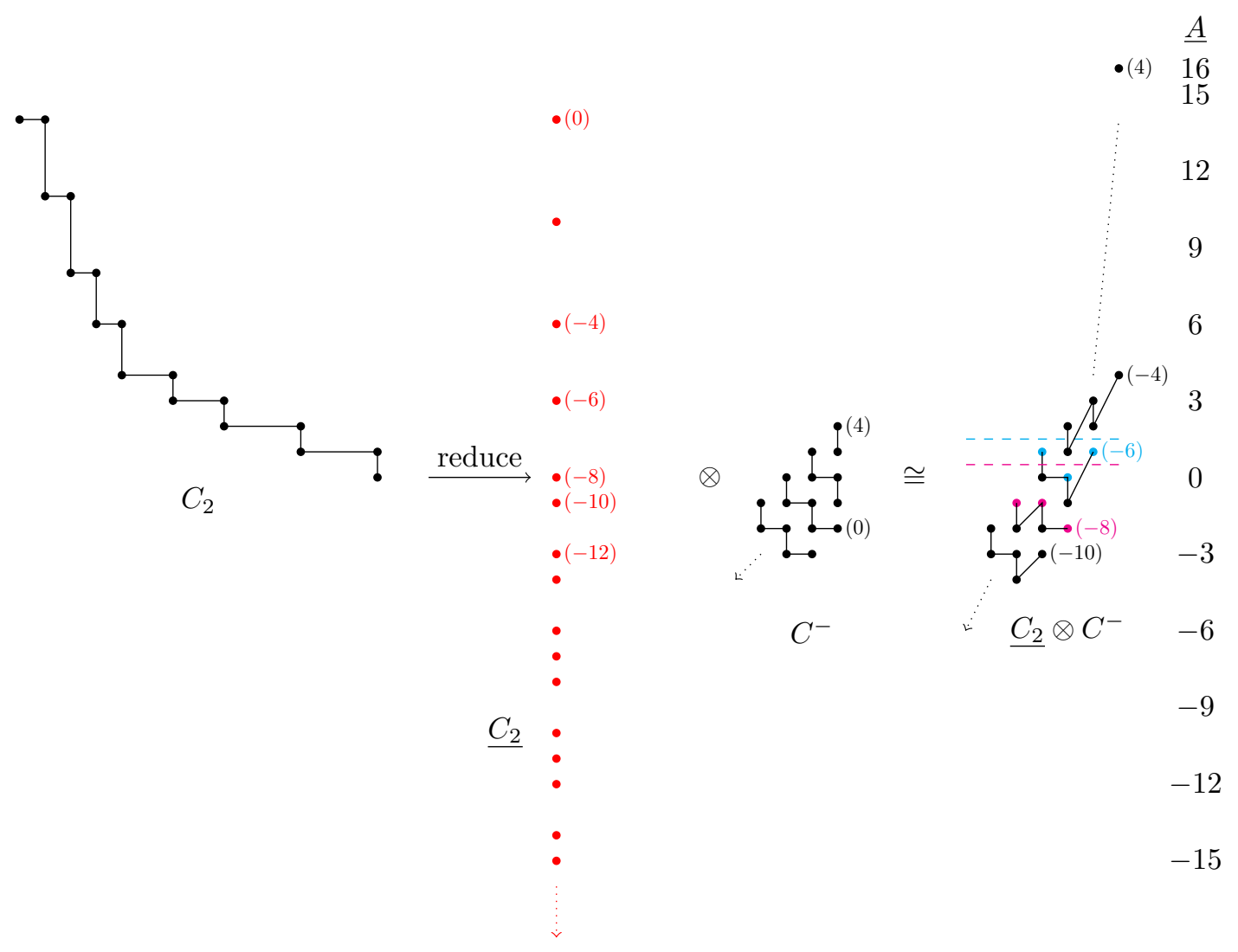

Figure 11. To the left is a generating set for the complex $C_{2}$. Reducing as in Figure 7 gives the red complex $C_{2}$. Tensoring this with $C^{-}$yields the result on the right (some of the complex which is not relevant to the computations at hand has been dotted out). The homological gradings of selected generators are given in parentheses. The element colored magenta - the highest grading generator of homology below the magenta line - demonstrates that $V_{0}=-\frac{1}{2}(-8)=4$, while the element colored blue demonstrates that $V_{1}=-\frac{1}{2}(-6)=3$.

Now we wish to compute $V_{0}$ and $V_{1}$ for the complex $C_{n} \otimes C^{-}$, which is easily done using the methods in the proof of [Krc15, Theorem 4.7]. To that end, let $\underline{C_{n}}$ be the reduced complex obtained from $C_{n}$ as described in Section 3.4. As a graded complex,

$$
\underline{C_{n}} \cong \mathbb{F}[U]_{(0)},
$$

but the filtration depends on the staircase; see Remark 3.16 . In the case at hand, the filtration level $A$ is given by

$$
A\left(U^{i} a\right)= \begin{cases}8 n-2-4 i & 0 \leq i \leq 2 n-2 \\ 3 & i=2 n-1 \\ 0 & i=2 n \\ -1 & i=2 n+1 \\ -3 & i=2 n+2 \\ -4 & i=2 n+3 \\ 2 n-i-2-\left\lfloor\frac{i-2 n-4}{3}\right\rfloor & 2 n+4 \leq i \leq 8 n-2 \\ -i & i \geq 8 n-2,\end{cases}
$$


where $a$ is the generator. Recall that $U$ drops grading by 2 , so that $M\left(U^{i} a\right)=-2 i$.

Now we consider the complex $\underline{C_{n}} \otimes C^{-}$. This complex is generated as an $\mathbb{F}[U]$-module by $a x_{j}$, for $1 \leq j \leq 5$. It is still filtered, but multiplication by $U$ is not homogeneous with respect to the filtration, precisely because it is not in $\underline{C_{n}}$. If we let $A$ be the filtration on $\underline{C_{n}}$ as given in $(3.3), A^{-}$ be the filtration on $C^{-}$, and $A^{\otimes}$ be the filtration on the product, then

$$
A^{\otimes}\left(U^{i} a x_{j}\right)=A\left(U^{i} a\right)+A^{-}\left(x_{j}\right) .
$$

Note that, in general, to get a filtration on a tensor product, we would set

$$
A^{\otimes}\left(U^{i} a x_{j}\right)=\min _{0 \leq k \leq i}\left\{A\left(U^{k} a\right)+A^{-}\left(U^{i-k} x_{j}\right)\right\} .
$$

Because $U$ lowers $A^{-}$by 1 and $U$ lowers $A$ by at least 1 , this is given by (3.4).

Remark 3.21. The homogeneous elements in $C_{n} \otimes C^{-}$with homological grading $-2 i$ are precisely $U^{i+2} a x_{1}, U^{i+1} a x_{3}$, and $U^{i} a x_{5}$. Further, $H_{-2 i}\left(\overline{C_{n}} \otimes C^{-}\right)$is generated by the sum of these elements. It follows from this and (3.4) that the filtration level of the generator of $H_{-2 i}$ is given by

$$
\max \left\{2+A\left(U^{i+2} a\right), A\left(U^{i+1} a\right),-2+A\left(U^{i} a\right)\right\} .
$$

Now we wish to determine the $V_{k}$ 's, recalling that $\underline{C_{n}} \otimes C^{-}$is $\nu^{+}$-equivalent to $2 J_{n}$. Note that

$$
\max \left\{2+A\left(U^{2 n+2} a\right), A\left(U^{2 n+1} a\right),-2+A\left(U^{2 n} a\right)\right\}=-1,
$$

while

$$
\max \left\{2+A\left(U^{2 n+1} a\right), A\left(U^{2 n} a\right),-2+A\left(U^{2 n-1} a\right)\right\}=1 .
$$

That is, the filtration level of the generator of $H_{-4 n}$ is -1 , and the filtration level of the generator of $H_{-4 n+2}$ is 1 . Together with 3.2 , these give that $V_{0}\left(2 J_{n}\right)=-\frac{1}{2}(-4 n)=2 n$, and $V_{1}\left(2 J_{n}\right) \leq 2 n-1$. By Proposition 3.3. $V_{1}$ and $V_{0}$ can differ by at most 1 , so it follows that $V_{1}(K)=2 n-1$.

\section{Proof of Theorem $\mathbf{A}$}

The goal of this section is to prove Theorem A Let $n$ be a positive integer and $L_{n}$ be the link in Figure 1. In the introduction section, we observed that $L_{n}$ has unknotted components and $L_{n}$ is topologically concordant to the Hopf link for any integer $n$. It remains to prove that $L_{n}$ is not smoothly concordant to any 2-component link $J$ with trivial Alexander polynomial, and $L_{n}$ and $L_{m}$ are not smoothly concordant when $n \neq m$. Therefore, the following theorems imply Theorem A.

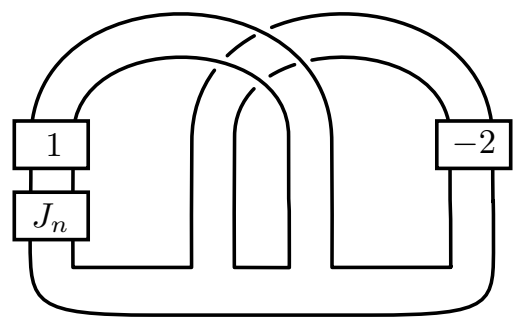

Figure 12. A disk-band form of a genus 1 Seifert surface of $K_{L_{n}}$.

Theorem 4.1. For any positive integer $n$, the link $L_{n}$ is not smoothly concordant to any 2-component link $J$ with trivial Alexander polynomial.

Proof. Throughout the proof, we continue to use the notations given in the statement of Theorem 2.8. Let $Z_{n}=\Sigma_{K_{L_{n}}}$ be the double cover of $Y_{L_{n}}$ branched along $K_{L_{n}}$. Suppose that $L_{n}$ is concordant to a link $J$ with trivial Alexander polynomial. By Theorem 2.8, there is a metabolizer $M$ for the linking form $\lambda_{Z_{n}}$ of $Z_{n}$ such that $\bar{d}\left(Z_{n}, \mathfrak{s}_{m}\right)=0$ for all $m$ in $M$.

Figure 12 gives a disk-band form of a genus 1 Seifert surface of $K_{L_{n}}$. Using the Akbulut-Kirby method [AK80, we can draw a surgery diagram of $Z_{n}$ as given in Figure 13. Let $\mu$ be a meridian of 


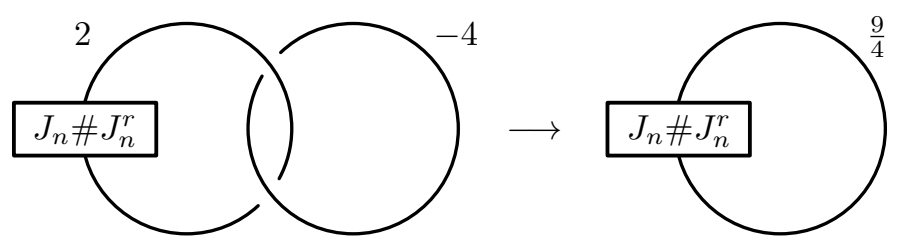

Figure 13. The 2-fold branched cover of $K_{L_{n}}$. The second figure is obtained by the slam-dunk move from the first figure.

the second figure of Figure $13 . H_{1}\left(Z_{n}\right)$ is generated by $\mu$ and $\lambda_{Z_{n}}(\mu, \mu)=-\frac{4}{9} \in \mathbb{Q} / \mathbb{Z}$. The linking form $\lambda_{Z_{n}}$ has unique metabolizer $M$ generated by $3 \mu$.

To obtain a contradiction, we show that $\bar{d}\left(Z_{n}, \mathfrak{s}_{3 \mu}\right) \neq 0$. Since $Z_{n}=S_{9 / 4}^{3}\left(J_{n} \# J_{n}^{r}\right)$, the Spin structure $\mathfrak{s}_{0}$ corresponds to the $\operatorname{Spin}^{c}$ structure $\mathfrak{t}_{6}$ by Remark 3.2 . We now observe that $\mathfrak{s}_{3 \mu}=\mathfrak{t}_{0}$. By the definition of $\mathfrak{s}_{3 \mu}$ and Remark 3.2 .

$$
\mathfrak{s}_{3 \mu}=\mathfrak{s}_{0}+3 \widehat{\mu}=\mathfrak{t}_{6}+3 \widehat{\mu}=\mathfrak{t}_{6}+3 \cdot 7 \widehat{\mu}=\mathfrak{t}_{0} .
$$

Further, using the recursive formula from [OS03, Section 4], it can be easily verified that $d\left(S_{9 / 4}^{3}(U), \mathfrak{t}_{0}\right)=$ $d\left(S_{9 / 4}^{3}(U), \mathfrak{t}_{6}\right)=0$.

By Proposition 3.1 and Theorem C, we have

$$
\begin{aligned}
\bar{d}\left(Z_{n}, \mathfrak{s}_{3 \mu}\right) & =d\left(Z_{n}, \mathfrak{s}_{3 \mu}\right)-d\left(Z_{n}, \mathfrak{s}_{0}\right) \\
& =d\left(S_{9 / 4}^{3}\left(J_{n} \# J_{n}^{r}\right), \mathfrak{t}_{0}\right)-d\left(S_{9 / 4}^{3}\left(J_{n} \# J_{n}^{r}\right), \mathfrak{t}_{6}\right) \\
& =d\left(S_{9 / 4}^{3}(U), \mathfrak{t}_{0}\right)-2 V_{0}\left(J_{n} \# J_{n}^{r}\right)-d\left(S_{9 / 4}^{3}(U), \mathfrak{t}_{6}\right)+2 V_{1}\left(J_{n} \# J_{n}^{r}\right) \\
& =2 V_{1}\left(J_{n} \# J_{n}^{r}\right)-2 V_{0}\left(J_{n} \# J_{n}^{r}\right) \\
& =2 V_{1}\left(2 J_{n}\right)-2 V_{0}\left(2 J_{n}\right)=4 n-2-4 n=-2,
\end{aligned}
$$

where in the last line we use the fact that $C F K^{\infty}\left(K^{r}\right) \cong C F K^{\infty}(K)$ OS04a, Proposition 3.9].

Theorem 4.2. If $n$ and $m$ are distinct positive integers, then the links $L_{n}$ and $L_{m}$ are not smoothly concordant.

Proof. We continue to use the notations used in Theorem 4.1. Suppose that $L_{n}$ and $L_{m}$ are smoothly concordant. The proof of Theorem 2.8 shows that there is a $\mathbb{Z}_{2}$-homology cobordism $W$ between $Z_{n}$ and $Z_{m}$. It follows that $d\left(Z_{n}, \mathfrak{s}_{0}\right)=d\left(Z_{m}, \mathfrak{s}_{0}\right)$. By the proof of Theorem 4.1. we have that $d\left(Z_{n}, \mathfrak{s}_{0}\right)=4 n$ and $d\left(Z_{m}, \mathfrak{s}_{0}\right)=4 m$ since $n, m \geq 1$. It follows that $n=m$. This completes the proof.

\section{References}

[AK80] S. Akbulut and R. Kirby, Branched covers of surfaces in 4-manifolds, Math. Ann. 252 (1979/80), no. 2, $111-131$.

[BCG17] J. Bodnár, D. Celoria, and M. Golla, A note on cobordisms of algebraic knots, Algebr. Geom. Topol. 17 (2017), no. 4, 2543-2564.

[BL14] M. Borodzik and C. Livingston, Heegaard Floer homology and rational cuspidal curves, Forum Math. Sigma 2 (2014), e28.

[CFP14] J. C. Cha, S. Friedl, and M. Powell, Concordance of links with identical Alexander invariants, Bull. Lond. Math. Soc. 46 (2014), no. 3, 629-642.

[CG88] T. D. Cochran and R. E. Gompf, Applications of Donaldson's theorems to classical knot concordance, homology 3-spheres and property P, Topology 27 (1988), no. 4, 495-512.

[CH15] T. D. Cochran and P. D. Horn, Structure in the bipolar filtration of topologically slice knots, Algebr. Geom. Topol. 15 (2015), no. 1, 415-428.

[CHH13] T. D. Cochran, S. Harvey, and P. D. Horn, Filtering smooth concordance classes of topologically slice knots, Geom. Topol. 17 (2013), no. 4, 2103-2162.

[CKRS12] J. C. Cha, T. Kim, D. Ruberman, and S. Strle, Smooth concordance of links topologically concordant to the Hopf link, Bull. Lond. Math. Soc. 44 (2012), no. 3, 443-450. 
[Dav06] J. F. Davis, A two component link with Alexander polynomial one is concordant to the Hopf link, Math. Proc. Cambridge Philos. Soc. 140 (2006), no. 2, 265-268.

[DR17] C. W. Davis and A. Ray, A new family of links topologically, but not smoothly, concordant to the Hopf link, J. Knot Theory Ramifications 26 (2017), no. 2, 1740002.

[DV16] A. Donald and F. Vafaee, A slicing obstruction from the $\frac{10}{8}$ theorem, Proc. Amer. Math. Soc. 144 (2016), no. $12,5397-5405$.

[End95] H . Endo, Linear independence of topologically slice knots in the smooth cobordism group, Topology Appl. 63 (1995), no. 3, 257-262.

[FQ90] M. H. Freedman and F. Quinn, Topology of 4-manifolds, Princeton Mathematical Series, vol. 39, Princeton University Press, Princeton, NJ, 1990.

[Fre82a] M. H. Freedman, A surgery sequence in dimension four; the relations with knot concordance, Invent. Math. 68 (1982), no. 2, 195-226.

[Fre82b] ㄴ. The topology of four-dimensional manifolds, J. Differential Geom. 17 (1982), no. 3, $357-453$.

[GM16] M. Golla and M. Marengon, Correction terms and the non-orientable slice genus, arXiv:1607.08117, 2016.

[Gom86] R. E. Gompf, Smooth concordance of topologically slice knots, Topology 25 (1986), no. 3, 353-373.

[GT04] S. Garoufalidis and P. Teichner, On knots with trivial Alexander polynomial, J. Differential Geom. 67 (2004), no. 1, 167-193.

[Hed09] M. Hedden, On knot Floer homology and cabling. II, Int. Math. Res. Not. IMRN (2009), no. 12, $2248-2274$.

[Hil02] J. Hillman, Algebraic invariants of links, Series on Knots and Everything, vol. 32, World Scientific Publishing Co. Inc., River Edge, NJ, 2002.

[HKL16] M. Hedden, S. G. Kim, and C. Livingston, Topologically slice knots of smooth concordance order two, J. Differential Geom. 102 (2016), no. 3, 353-393.

[HLR12] M. Hedden, C. Livingston, and D. Ruberman, Topologically slice knots with nontrivial Alexander polynomial, Adv. Math. 231 (2012), no. 2, 913-939.

[HM17] K. Hendricks and C. Manolescu, Involutive Heegaard Floer homology, Duke Math. J. 166 (2017), no. 7, 1211-1299.

[Hom14] J. Hom, The knot Floer complex and the smooth concordance group, Comment. Math. Helv. 89 (2014), no. 3, 537-570.

[Hom15] _ An infinite-rank summand of topologically slice knots, Geom. Topol. 19 (2015), no. 2, 1063-1110.

[Hom17] _ A survey on Heegaard Floer homology and concordance, J. Knot Theory Ramifications 26 (2017), no. 2,1740015 .

[HW14] M. Hedden and L. Watson, On the geography and botany of knot Floer homology, arXiv:1404.6913, 2014.

[HW16] J. Hom and Z. Wu, Four-ball genus bounds and a refinement of the Ozváth-Szabó tau invariant, J. Symplectic Geom. 14 (2016), no. 1, 305-323.

[Kaw96] A. Kawauchi, A survey of knot theory, Birkhäuser Verlag, Basel, 1996.

[KP16] M. H. Kim and K. B. Park, An infinite-rank summand of knots with trivial Alexander polynomial, arXiv:1604.04037, to appear in J. Symplectic Geom., 2016.

[Krc15] D. Krcatovich, The reduced knot Floer complex, Topology Appl. 194 (2015), 171-201.

[Liv08] C. Livingston, Slice knots with distinct Ozsváth-Szabó and Rasmussen invariants, Proc. Amer. Math. Soc. 136 (2008), no. 1, 347-349.

[MO07] C. Manolescu and B. Owens, A concordance invariant from the Floer homology of double branched covers, Int. Math. Res. Not. IMRN (2007), no. 20, 21 pages.

[NW15] Y. Ni and Z. Wu, Cosmetic surgeries on knots in $S^{3}$, J. Reine Angew. Math. 706 (2015), 1-17.

[OS03] P. Ozsváth and Z. Szabó, Absolutely graded Floer homologies and intersection forms for four-manifolds with boundary, Adv. Math. 173 (2003), no. 2, 179-261.

[OS04a] _ Holomorphic disks and knot invariants, Adv. Math. 186 (2004), no. 1, 58-116.

[OS04b] Holomorphic disks and topological invariants for closed three-manifolds, Ann. of Math. (2) 159 (2004), no. 3, 1027-1158.

[OS05] On knot Floer homology and lens space surgeries, Topology 44 (2005), no. 6, 1281-1300.

[OS08] _ Knot Floer homology and integer surgeries, Algebr. Geom. Topol. 8 (2008), no. 1, 101-153.

[OS11] Knot Floer homology and rational surgeries, Algebr. Geom. Topol. 11 (2011), no. 1, 1-68.

[OSS17] P. Ozsváth, A. Stipsicz, and Z. Szabó, Concordance homomorphisms from knot Floer homology, Adv. Math. 315 (2017), 366-426.

[Ras03] J. A. Rasmussen, Floer homology and knot complements, ProQuest LLC, Ann Arbor, MI, 2003, Thesis (Ph.D.)-Harvard University.

[Tor53] G. Torres, On the Alexander polynomial, Ann. of Math. (2) 57 (1953), 57-89. 